\title{
The structure of diffraction and the diffractive hadronic final states in $e p$ collisions at HERA
}

\author{
Anatoli Astvatsatourov a \\ ${ }^{a}$ I.I.H.E., Vrije Universiteit Brussel, Belgium
}

Recent measurements of diffractive processes at HERA, which test the QCD factorization in high energy diffractive DIS (DDIS) and photoproduction $\left(\gamma^{*} p\right)$ hard scale processes are compared to leading order (LO) and next to leading order (NLO) predictions. QCD factorization is seen to hold for $D^{*}$ production, both in DDIS and photoproduction, as well as for dijets in DDIS, but fails for dijets in photoproduction.

\section{Introduction}

The data measured in e $p$ collisions at HERA contain about $10 \%$ of diffractive events $[1,2]$, when a large gap in rapidity relative to the hadronic final state is observed. In most cases, in these diffractive events, the proton survives the collision intact, keeping a significant part of its initial energy, i.e. the four-momentum transfer at the proton vertex $t$ is very small.

In Regge framework these diffractive events can be described in terms of a pomeron $(\mathbb{P})$ exchange $[3,4]$. Here the pomeron is a colour-singlet hadronic component of the proton carrying a fraction of the proton momentum $x_{\mathbb{P}}$. Due to the presence of a hard scale in diffractive interactions, the parton densities of the pomeron can be extracted from DDIS data at HERA, assuming Regge and QCD factorization (see section 2).

However, these diffractive parton densities extracted under the assumption of QCD factorization are unable to reproduce the diffractive data at the Tevatron [5-7]. Alternative approaches based on the soft colour exchanges, which have been developed in [28-30], are mentioned in section 7 .

\section{Factorization and diffractive parton dis- tributions}

The QCD factorization theorem [8] which has been proven for DDIS processes [9] implies that the cross section of diffractive processes can be written in terms of the Parton Distribution Func- tions (PDF), where the PDF's $f_{i, \mathrm{P}}^{D}$ represents a conditional probability distribution of parton in the proton under the constraint of a leading final state proton:

$\sigma^{D}=\sum_{i} f_{i, \mathbb{P}}^{D} \otimes \sigma^{\gamma, i}$

An additional Regge factorization assumption, as applied in the Ingelman-Schlein (IS) or resolved pomeron model [10], assumes that the diffractive PDF's (dPDF) can be factorized into a flux factor depending only on $x_{\mathbb{P}}$ and $t$ :

$$
\begin{aligned}
& f_{i, \mathbb{P}}^{D(4)}\left(x_{B j}, Q^{2}, x_{\mathbb{P}}, t\right)= \\
& f_{\mathbb{P} / p}\left(x_{\mathbb{P}}, t\right) \cdot p_{i, \mathbb{P}}\left(\beta, Q^{2}\right)
\end{aligned}
$$

Here $x_{B j}$ is the Bjorken scaling variable, $\beta$ the fraction of $\mathbb{P}$-momentum carried by a struck quark $\left(\beta=x_{B j} / x_{\mathbb{P}}\right), p_{i, \mathbb{P}}$ the $\mathbb{P}$-parton density and $f_{\mathbb{P} / p}$ the $\mathbb{P}$-flux factor.

If factorization of equation (1) holds and an assumption (2) is valid, then one may measure the pomeron parton densities $p_{i, \mathbb{P}}$ from inclusive DDIS data and use them to predict cross sections of other diffractive processes.

\section{Pomeron Parton Densities}

The diffractive parton densities $p_{i, \mathbb{P}}$ composed of a light flavour singlet and gluon density as extracted from the measurements are shown in Fig. 1 as a function of $z$, where $z$ is the momentum fraction of the parton entering the hard subprocess with respect to the diffractive exchange. 
For the lowest order quark parton model process $z=\beta$, whereas for higher order processes $0<\beta<z$.

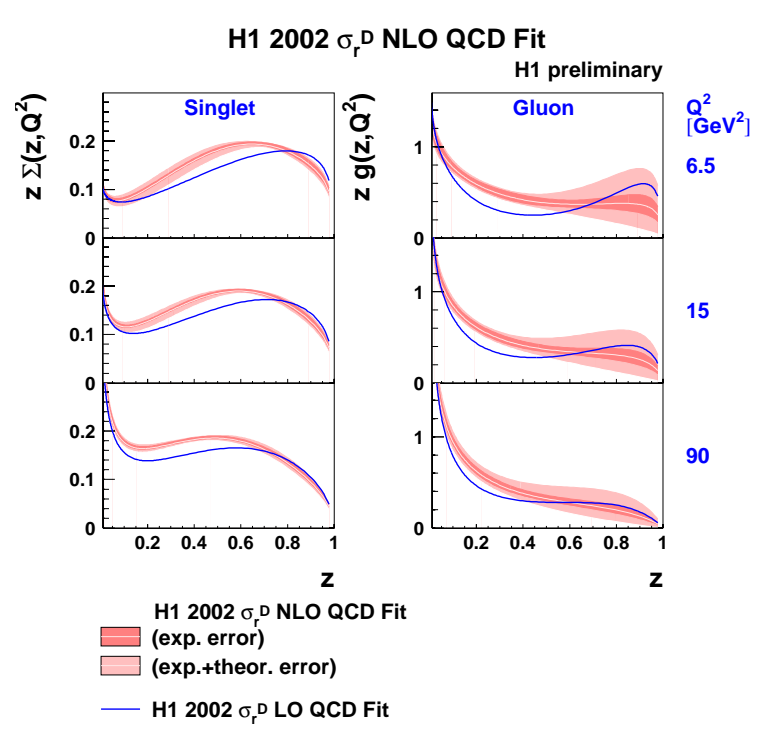

Figure 1. Quark singlet and gluon distributions in three $Q^{2}$ bins, obtained using the DGLAP [11] equations both in $\mathrm{LO}$ and NLO.

The inner and outer error bands represent respectively the experimental error and experimental and theoretical errors added in quadrature. The shape of the dPDF's in the fit are assumed to be independent of $x_{\mathbb{P}}$. The momentum fraction carried by the gluons in a diffractive exchange amounts to about $75 \%[12,13]$.

\section{Diffractive $\mathrm{D}^{*}$ mesons in DIS}

Diffractive $D^{*}$ at HERA are predominantly produced via photon-gluon fusion $\gamma g \rightarrow c \bar{c}$. The $D^{*}$ mesons are reconstructed from the decay channel:

$D^{*} \rightarrow D^{o} \pi_{s} \rightarrow K \pi \pi_{s}$

where $\pi_{s}$ is a lowest momentum $\pi$-meson in the $D^{*}$ decay.

Recent results of diffractive $D^{*}$ production in DIS by the H1 and ZEUS experiments [14-17] agree well with the NLO prediction (see Fig. 2) calculated using the dPDF's described in sections 2 and 3.

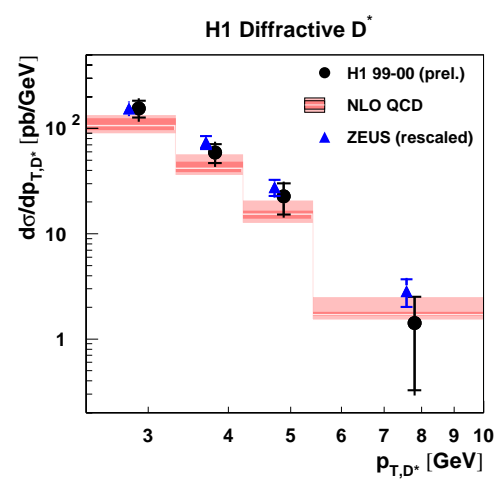

Figure 2. Differential cross section for diffractive $D^{*}$ meson production in DIS. The data are shown as dots with inner and outer error bars presenting statistical and total errors respectively. The data are compared to a NLO QCD calculation.

\section{5. $\mathrm{D}^{*}$ mesons in photoproduction}

Diffractive photoproduction of $D^{*}$ mesons has been recently measured with the ZEUS detector at HERA [27]. The $D^{*}$ mesons have been reconstructed from the decay channel of equation 3 .
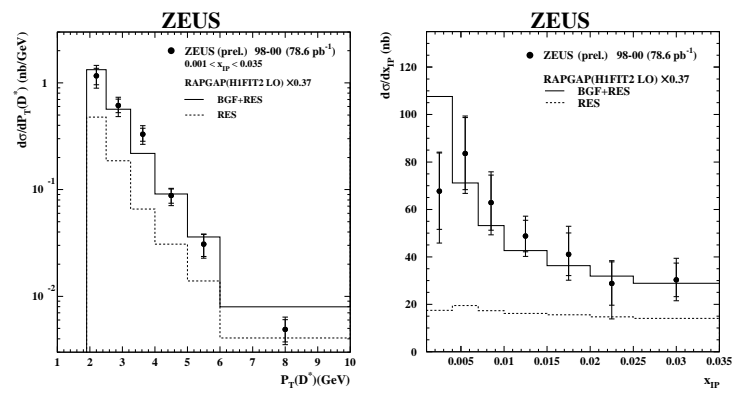

Figure 3. The differential cross sections $d \sigma / d p_{t}^{D^{*}}$ and $d \sigma / d x_{\mathbb{P}}$ measured at the ZEUS detector for the $e p \rightarrow e D^{*} X p$ diffractive photoproduction.

The cross sections shown in Fig. 3 are compared to the LO (RAPGAP) prediction. The solid histogram shows the LO theoretical cross section 
normalized to the data. The shape of data is well described by the LO Monte Carlo. The predicted contribution from the resolved photon reactions is shown as the dashed histogram.

\section{Dijets in DIS and in photoproduction}

The differential cross section of dijets in DIS as a function of $z_{\mathbb{P}}$ and $\log _{10}\left(x_{\mathbb{P}}\right)$ measured at H1 is compared to LO (RAPGAP [18]) and NLO (DISENT [19]) predictions and shown in Fig. 4.

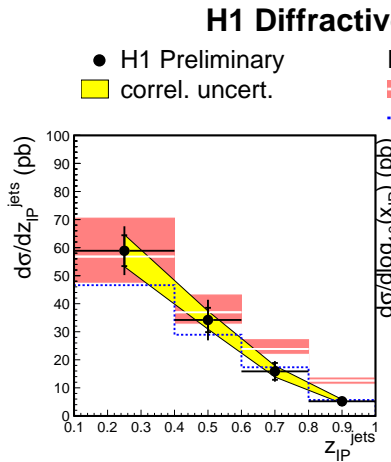

(a)

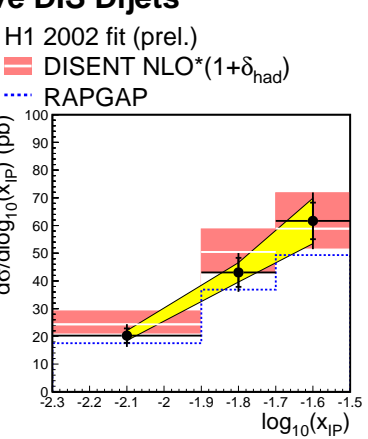

(b)
Figure 4 . The differential cross sections $d \sigma / d z_{\mathbb{P}}$ and $d \sigma / d \log _{10}\left(x_{\mathbb{P}}\right)$ of dijets in DIS measured at the $\mathrm{H} 1$ detector.

The NLO prediction is in a good agreement with the $\mathrm{H} 1$ data for $z_{\mathrm{PP}}^{\text {jets }}<0.6$. At higher values the predictions overestimate the measured cross section [20]. The ZEUS data are compared to the "NLO-LPS" and to the "H1 2002 fit" NLO predictions. The NLO prediction has a tendency to be higher than the data at high $Q^{2}$ and $\beta$ [21], but the factorization holds for dijets in DIS.

The differential cross section of dijets in photoproduction as a function of $z_{\mathbb{P}}$ and $x_{\gamma}$ is compared to the LO and NLO predictions and shown in Fig. 5. The cross section measured at H1 $[20,24]$ agrees with the ZEUS data $[22,23]$ and shows that the NLO prediction overestimates the dijets photoproduction cross section by a factor $\sim 2$.

The measured cross section of dijets in photoproduction shows quantitative disagreement to the theoretical prediction $[25,26]$.
QCD factorization fails in this process both for direct $\left(x_{\gamma}>0.75\right)$ and resolved $\left(x_{\gamma}<0.75\right)$ components, where $x_{\gamma}$ is the fraction of the photon momentum involved in the hard subprocess. The ratio of data to NLO prediction is flat for both direct and resolved components.

The results shown in Figures 4 and 5 for dijet in DIS and in photoproduction not include the diffractive PDF uncertainty.

\section{H1 Diffractive yp Dijets}

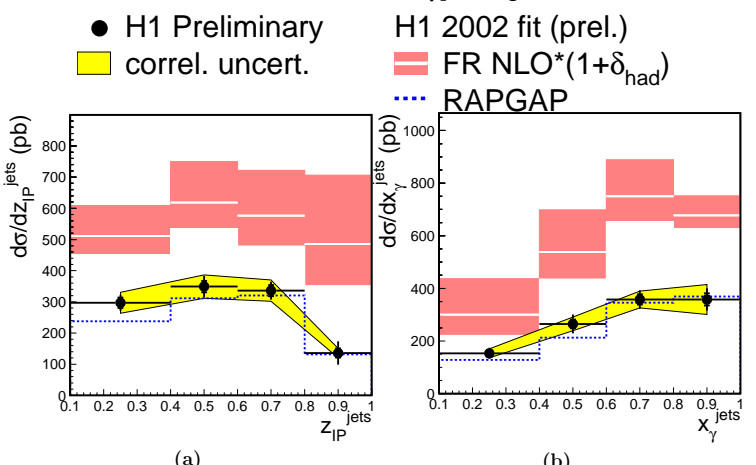

Figure 5. The differential cross section of dijets in photoproduction measured at H1.

The NLO error bands in Figures 4,5 indicate the uncertainties in the hadronization corrections and renormalization scale [20]. The diffractive PDF uncertainty is not included in the NLO calculation.

\section{Approaches to factorization breaking}

Other approaches to describe diffractive phenomena have been proposed in SCI [28] and BHMPS [29] models. In these models events with a large rapidity gap are the result of a soft rescattering, where a "target gluon" with momentum fraction of $x_{\mathbb{P}}$ splits into $q \bar{q}$ or $g g$ pair and the virtual $\gamma$ is absorbed on one of the partons in the pair, or scattered on a quark dipole via $\gamma^{*} g \rightarrow Q \bar{Q} g$. Then, before hadronization, the colour octet $(q \bar{q})$ or $(Q \bar{Q} g)$ is turned to a colour singlet by soft longitudinal gluon exchange.

The diffractive parton distributions in these rescattering models are process dependent be- 
cause of different colour structures in $\gamma p$ and $p \bar{p}$ interactions and because of the constraint to have a colour singlet in the final state [30].

\section{Conclusion}

LO and NLO QCD fits have been performed to diffractive data at HERA to test the validity of the QCD factorization theorem for diffractive dijets and $D^{*}$ production in DIS and photoproduction regimes. Recent results from $\mathrm{H} 1$ and ZEUS show that the factorization holds in DDIS scattering and fails for dijets $\gamma^{*} p$ photoproduction as shown in Fig. 6.

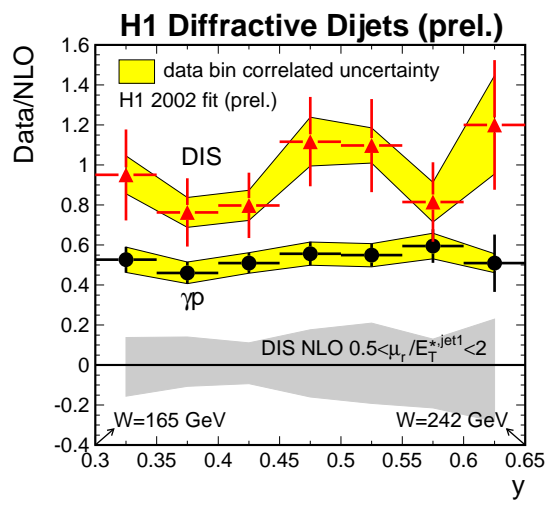

Figure 6. Ratio of data to NLO prediction for diffractive dijets in DIS and in $\gamma^{*} p$ photoproduction regimes as a function of inelastisity $y$.

The $D^{*}$ mesons in diffractive photoproduction at HERA are measured at ZEUS detector and a comparison with the NLO prediction is done in the mean time. The factorization holds in this process.

\section{REFERENCES}

1. ZEUS coll., Phys. Lett. B 315, 481 (1993).

2. H1 coll., Nucl. Phys. B 429, 477 (1994).

3. M. Wüsthoff and A.D. Martin, J. Phys. G 25, 309 (1999) [hep-ph/9909362].

4. J.Bartels, J.R.Ellis, H.Kowalski and M.Wüsthoff, Eur. Phys. J. C 7, 443 (1999) [hep-ph/9803497].

5. CDF coll., Phys. Rev. Lett. 79, 2636 (1997).
6. CDF coll., Phys. Rev. Lett. 85, 4215 (2000).

7. D0 coll., Phys. Lett. B 531, 52 (2002)

8. J.C. Collins and D.E. Soper, Nucl. Phys. B 194, 445 (1982); J.C. Collins, D.E. Soper, G.Sterman, Nucl. Phys. B 261, 104 (1985); [hep-ph/9806234].

9. J.C.Collins, Phys.Rev. D 57 (1998) 3051 and erratum-ibid. D 61019902 (2000).

10. G.Ingelman and P.Schlein, Phys. Lett. B 152, 256 (1985).

11. V.N.Gribov and L.N.Lipatov, Sov. J. Nucl. Ph. 15, 438 (1972);

L.N.Lipatov, Sov. J. Nucl. Ph. 20, 94 (1975); Yu.L.Dokshitzer, Sov. JETP 46, 641 (1977); G.Altarelli and G.Parisi, Nucl. Phys. B 126, 298 (1977).

12. H1 coll., Z. Phys. C 76, 613 (1997).

13. H1 coll., ICHEP2002, abstract: 980.

14. H1 coll., ICHEP04, abstract: 6-0178.

15. H1 coll. [hep-ex/0503038], DESY-05-040.

16. ZEUS coll., Lepton-Photon 2005, abst.: 271.

17. ZEUS coll., Lepton-Photon 2005, abst.: 266.

18. H. Jung, Comp. Phys. Commun. 86147 (1995).

19. S. Catani, M.H. Seymour, CERN-TH/96-240 [hep-ph/9609521]

20. H1 coll., ICHEP04, abstract: 6-0177.

21. ZEUS coll., Lepton-Photon 2005, abst.: 295.

22. ZEUS coll., ICHEP04, abstract: 6-0249; ZEUS-prel-05-001, Talk on "HERA and the LHC Final Meeting", DESY, March 21-24 2005.

23. ZEUS coll., DIS05, Proceedings, p494.

24. H1 coll., DIS05, Proceedings., p488.

25. A.B. Kaidalov, V.A. Khoze, A.D. Martin and M.G. Ryskin, Eur. Phys. J. C 21, 521 (2001) [hep-ph/0105145].

26. A.B. Kaidalov, V.A. Khoze, A.D. Martin and M.G. Ryskin, Phys. Lett. B 567, 61 (2003).

27. ZEUS coll., Lepton-Photon 2005, abst.: 268.

28. A.Edin, G.Ingelman and J.Rathsman, Phys. Lett. B 366, 371 (1996) [hep-ph/9508386]; Z. Phys. C 75, 57 (1997) [hep-ph/9605281].

29. S.J.Brodsky, P.Hoyer, N.Marchal, S.Peigné and F.Sannino, Phys. Rev. D 65, 114025 (2002) [hep-ph/0104291].

30. S.J.Brodsky, R.Enberg, P.Hoyer and G.Ingelman, SLAC-PUB-10692 [hep-ph/0409119]. 